OPEN ACCESS

Edited by:

Megan Anne Cooper,

Washington University in St. Louis,

United States

Reviewed by:

Elie Haddad,

Université de Montréal, Canada

Jeffrey J. Bednarski,

Washington University School of

Medicine in St. Louis, United States

*Correspondence: Nirali N. Shah

Nirali.Shah@nih.gov

Specialty section:

This article was submitted to

Primary Immunodeficiencies,

a section of the journal

Frontiers in Immunology

Received: 25 October 2021 Accepted: 01 December 2021 Published: 17 December 2021

Citation:

Holland EM, Gonzalez C, Levy E, Valera VA, Chalfin H, Klicka-Skeels J,

Yates B, Kleiner DE, Hadigan C,

Dave H, Shalabi H, Hickstein DD,

Su HC, Grimley M, Freeman AF and Shah NN (2021) Case Report: Fatal

Complications of BK Virus-

Hemorrhagic Cystitis and Severe Cytokine Release Syndrome Following $B K$ Virus-Specific T-Cells.

Front. Immunol. 12:801281. doi: 10.3389/fimmu.2021.801281

\section{Case Report: Fatal Complications of BK Virus-Hemorrhagic Cystitis and Severe Cytokine Release Syndrome Following BK Virus-Specific T-Cells}

\author{
Elizabeth M. Holland ${ }^{1}$, Corina Gonzalez ${ }^{1,2}$, Elliot Levy ${ }^{3}$, Vladimir A. Valera ${ }^{4}$, \\ Heather Chalfin ${ }^{4}$, Jacquelyn Klicka-Skeels ${ }^{5}$, Bonnie Yates ${ }^{1}$, David E. Kleiner ${ }^{6}$, \\ Colleen Hadigan ${ }^{7}$, Hema Dave ${ }^{5}$, Haneen Shalabi ${ }^{1}$, Dennis D. Hickstein ${ }^{2}$, Helen C. Su ${ }^{8}$, \\ Michael Grimley ${ }^{9}$, Alexandra F. Freeman ${ }^{8}$ and Nirali N. Shah ${ }^{1 *}$

\begin{abstract}
Pediatric Oncology Branch, Center for Cancer Research (CCR), National Cancer Institute (NCI), National Institutes of Health (NIH), Bethesda, MD, United States, ${ }^{2}$ Immune Deficiency-Cellular Therapy Program, Center for Cancer Research (CCR), National Cancer Institute (NCI), National Institutes of Health (NIH), Bethesda, MD, United States, ${ }^{3}$ Radiology and Imaging Sciences, NIH Clinical Center (CC), Bethesda, MD, United States, ${ }^{4}$ Urologic Oncology Branch, Center for Cancer Research (CCR), National Cancer Institute (NCI), National Institutes of Health (NIH), Bethesda, MD, United States, 5 Pediatric Oncology, Children's National Medical Center, Washington, DC, United States, ${ }^{6}$ Laboratory of Pathology, National Cancer Institute (NCl), National Institutes of Health (NIH), Bethesda, MD, United States, ${ }^{7}$ Pediatric Gastroenterology, NIH Clinical Center (CC), Bethesda, MD, United States, ${ }^{8}$ Laboratory of Clinical Immunology and Microbiology, National Institutes of Allergy and Infectious Disease, NIH Clinical Center (CC), Bethesda, MD, United States, ${ }^{9}$ Division of Bone Marrow Transplantation and Immune Deficiency, Cincinnati Children's Hospital, Cincinnati, $\mathrm{OH}$, United States
\end{abstract}

BK virus (BKV)-hemorrhagic cystitis $(\mathrm{HC})$ is a well-known and rarely fatal complication of hematopoietic stem cell transplantation (HSCT). Treatment for BKV-HC is limited, but virus-specific T-cells (VST) represent a promising therapeutic option feasible for use posttransplant. We report on the case of a 16-year-old male with dedicator of cytokinesis 8 (DOCK8) deficiency who underwent haploidentical HSCT complicated by severe BKV$\mathrm{HC}$, catastrophic renal hemorrhage, and VST-associated cytokine release syndrome (CRS). Gross hematuria refractory to multiple interventions began with initiation of posttransplant cyclophosphamide (PT/Cy). Complete left renal arterial embolization (day +43 ) was ultimately indicated to control intractable renal hemorrhage. Subsequent infusion of anti-BK VSTs was complicated by CRS and progressive multiorgan failure, with postmortem analysis confirming diagnosis of hepatic sinusoidal obstruction syndrome (SOS). This case illustrates opportunities for improvement in the management of severe BKV-HC posttransplant while highlighting rare and potentially life-threatening complications of BKV-HC and VST therapy.

Keywords: DOCK8 immunodeficiency syndrome, HSCT = hematopoietic stem cell transplant, BK virus associated hemorrhagic cystitis, virus specific T-cells, cytokine release syndrome 


\section{INTRODUCTION}

Allogeneic hematopoietic stem cell transplantation (HSCT) represents the only curative therapy for reversal of the clinical and immunological phenotype of dedicator of cytokinesis 8 (DOCK8) deficiency (1-5), an autosomal recessive combined immunodeficiency characterized by eczematous dermatitis, sinopulmonary infections, allergy, susceptibility to DNA viral infections, elevated serum IgE, and cancer predisposition $(6,7)$. Risk of post-HSCT viral reactivation and complications thereof remains elevated in this disease (5) and may be especially high in patients undergoing HSCT conditioning with posttransplant cyclophosphamide (PT/Cy). BK virus (BKV)-hemorrhagic cystitis (HC) can contribute to significant morbidity following HSCT but is seldom associated with fatal complications. Treatment options for BKV-HC are limited. While complications of BKV-HC are rarely life-threatening, we report on and discuss the management of a patient with $\mathrm{BK}$ viremia whose post-HSCT course was uniquely complicated by severe BKV-HC with combined renal hemorrhage and significant cytokine release syndrome (CRS) following treatment with BKtargeted virus specific T-cells (VSTs).

\section{CASE}

A 16-year-old male with DOCK8 deficiency (homozygous for DOCK8 variant NM_203447.3:c.4153+1G>A) enrolled on an IRB-approved National Cancer Institute HSCT trial for patients with DOCK8 (NCT01176006). His disease, diagnosed at age 8 years, manifested with recurrent sinopulmonary infections, chronic molluscum contagiosum, and eczematous dermatitis. Recent complications included diagnosis of Diffuse Large B-Cell Lymphoma (DLBCL) 4 months pre-HSCT. Treatment with rituximab (4 doses) and LMB regimen $(8,9)$ (2 cycles), including vincristine, doxorubicin, corticosteroids, methotrexate and with cumulative cyclophosphamide dose of $3300 \mathrm{mg} / \mathrm{m}^{2}$ as per ANHL1131, Group B (R-COPADM), was complicated by chemotherapy-associated grade III BKV-HC managed with cidofovir and 2 infusions of third-party donorderived quadrivalent anti-cytomegalovirus (CMV), -EpsteinBarr virus (EBV), -adenovirus (ADV), and $-\mathrm{BK}$ virus specific T-cells (VSTs) at $5 \times 10^{7}$ cells $/ \mathrm{m}^{2}$ (NCT02532452) (10). VSTs were well tolerated without any infusion reaction. HC symptoms subsequently resolved while asymptomatic BK viremia persisted. Achieving a complete remission, he proceeded to HSCT with his father as the haploidentical bone marrow donor. Reduced

\footnotetext{
Abbreviations: ADV, adenovirus; AKI, acute kidney injury; BKV, BK virus; CMV, cytomegalovirus; CRP, C-reactive protein; CRRT, continuous renal replacement therapy; CRS, cytokine release syndrome; DLBCL, Diffuse large B-cell lymphoma; DOCK8, dedicator of cytokinesis 8; EBV, Epstein-Barr Virus; GVHD, graftversus-host disease; HC, hemorrhagic cystitis; HLA, human leukocyte antigen; HSCT, hematopoietic stem cell transplantation; LMB, Lymphomes Malins B therapy; PT/Cy, posttransplant cyclophosphamide; RIC, reduced intensity conditioning; SOS, sinusoidal obstruction syndrome; TBI, total body irradiation; VOD, veno-occlusive disease; VST, virus specific T-cell.
}

intensity (RIC) conditioning was comprised of fludarabine 30 $\mathrm{mg} / \mathrm{m}^{2} \times 5$ days (days -6 to -2 ), busulfan dosed with target area under the curve (AUC) of 3600-4000 uM.min/day x 3 days (days -4 to -2 ), cyclophosphamide $14.5 \mathrm{mg} / \mathrm{kg}$ x 2 days (days -6 and -5), and low-dose total body irradiation (TBI, $200 \mathrm{cGy}$ ) on day -1. Graft-versus-host disease (GVHD) prophylaxis was comprised of post-transplant cyclophosphamide (PT/Cy) on days +3 and +4 along with mycophenolate mofetil (MMF) x 30 days and tacrolimus x 6 months, both of which started on day +5 (11). Mild cystitis without hematuria developed on HSCT day 0 . BK viremia simultaneously increased from 588,844 copies/mL (3 days pre-HSCT) to $6,456,542$ copies $/ \mathrm{mL}$ on day +4 (Figure 1A).

His immediate post-HSCT course was complicated by gross hematuria and painful bladder spasms with PT/Cy $(50 \mathrm{mg} / \mathrm{kg})$ on days +3 and +4 . Grade IV gross hematuria, managed with daily blood product transfusions, persisted alongside worsening acute kidney injury (AKI, day +14) (Figure 1A). Continued transfusion dependence led to significant fluid retention and $11 \mathrm{~kg}$ weight gain over 11 days. Daily platelet infusions were used to maintain platelet count $\geq 30 \mathrm{~K} / \mathrm{mcL}$, and platelet engraftment $\geq 50 \mathrm{~K} / \mathrm{mcL}$ was not attained. Neutrophil engraftment was achieved at day +21 ; chimerism studies demonstrated $100 \%$ donor-derived cells without evidence of GVHD.

Progressive hydronephrosis and hydroureter prompted Foley catheter and bilateral nephrostomy tube placement by day +30 . Gross hematuria from the left nephrostomy tube occasioned left renal arteriography which revealed active bleeding unrelated to the nephrostomy at multiple sites and features suggestive of vasculitis. Coil embolization performed on two separate occasions (days +35 and +38 ) provided only transient stabilization. Ongoing bleeding and transfusion needs were accompanied by increasing total and direct hyperbilirubinemia $(2.9 \mathrm{mg} / \mathrm{dL}$ and $2.7 \mathrm{mg} / \mathrm{dL}$, respectively $($ day +43$))$. Complete left renal artery embolization on day +42 , followed by initiation of intravenous cidofovir (Figures 2A-H), stabilized the patient for transport to receive an additional infusion of third-party VSTs $($ day +43$)$ produced using the same donor as his second preHSCT infusion (NCT02532452). BK viremia measured 5,754,399 copies $/ \mathrm{mL}$ at third infusion, nearly a full log increase from viral load with pre-transplant VSTs (Figure 1B).

Two days after VST infusion (day +45), onset of cytokine release syndrome (CRS) was evidenced by fever, hypotension, worsening lung opacities, and bilateral pleural effusions. Inflammatory markers indicative of CRS were also elevated. CRP peaked at $268.7 \mathrm{mg} / \mathrm{L}$ on day +43 following VST infusion. Interestingly, CRP had been rising in the days leading up to VST infusion, potentially in the context of significant bleeding and interventional procedures. A rapid rise in ferritin was seen from $4,641 \mathrm{mcg} / \mathrm{mL}$ pre-VSTs $($ day +40$)$ to $5,313 \mathrm{mcg} / \mathrm{mL}$ postinfusion (day +45$)$ and $17,456 \mathrm{mcg} / \mathrm{mL}$ on day +47 (Figure 1C). Plasma IL-6 rose from $360 \mathrm{pg} / \mathrm{mL}$ pre-infusion $($ day +41$)$ to $2,182 \mathrm{pg} / \mathrm{mL}$ on day +45 with CRS onset and prior to initiation of the IL-6R (receptor) blocker tocilizumab (Figure 1D). Concurrent blood, urine, and viral testing remained negative for any signs of new infection. Aggressive 

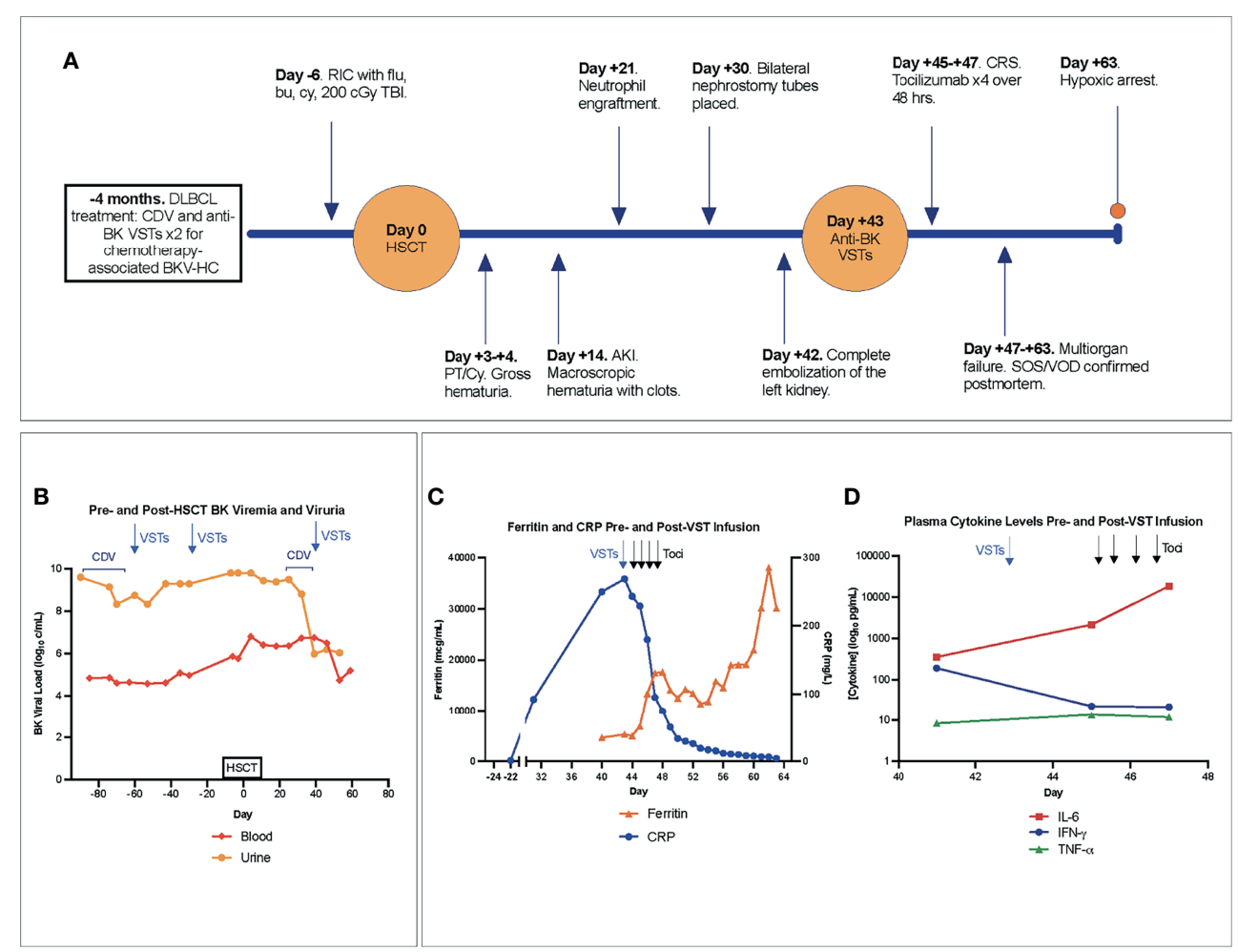

FIGURE 1 | (A) Timeline of major events pre- and post-HSCT. (B) BK viremia and viruria with therapeutic interventions denoted in the pre- and post-transplant period. (C) Blood ferritin, CRP, and (D) plasma cytokine levels pre- and post-onset of CRS, with arrows indicating VST infusion (blue) and delivery of each of 4 total doses of tocilizumab (black). Notably, the first dose of tocilizumab was administered after cytokine levels were drawn on day +45 . AKI, acute kidney injury; Bu, busulfan; CDV, cidofovir; CRP, C-reactive protein; CRS, cytokine release syndrome; Cy, cyclophosphamide; DLBCL, Diffuse large B-cell lymphoma; Flu, fludarabine; HSCT, hematopoietic stem cell transplantation; IFN- $\gamma$, interferon gamma; IL-6, interleukin-6; VST, quadrivalent anti-CMV, -EBV, -ADV, and -BK virus specific T-cells; PT/Cy, post-transplant cyclophosphamide; RIC, reduced intensity conditioning; SOS, sinusoidal obstructive syndrome; TBI, total-body irradiation; Toci, tocilizumab; TNF- $\alpha$, Tumor Necrosis Factor alpha; VOD, veno-occlusive disease.

fluid resuscitation, bilateral chest tube placement, vasopressor support, continuous renal replacement therapy (CRRT), and 4 doses of tocilizumab ( $8 \mathrm{mg} / \mathrm{kg})$ were given over 48 hours. Following fluid resuscitation for CRS, liver studies (day +47) demonstrated worsening hyperbilirubinemia. Liver ultrasound showed hepatosplenomegaly and sluggish flow through the main portal vein, raising concern for late-onset sinusoidal obstruction syndrome (SOS)/veno-occlusive disease (VOD). Given prior lifethreatening hemorrhage, defibrotide was contraindicated. Multiorgan failure and worsening coagulopathy led to hypoxic arrest on day +63 . Autopsy was declined but limited postmortem single core liver, kidney, and lung biopsies demonstrated hepatic SOS/VOD with zone 3 hemorrhagic necrosis, acute renal tubular injury, and early pulmonary exudative phase diffuse alveolar damage (Figures 2I-L). Limited tissue SV40 immunostain for polyomavirus was negative at all 3 sites.

\section{DISCUSSION}

Complications of post-transplantation viral reactivation remain a significant challenge in patients with DOCK8 deficiency due to the underlying immunodeficiency associated with DOCK8 mutation. We highlight unique challenges of managing BKVHC with hemorrhagic nephropathy and describe potential complications of anti-BK VST therapy.

Inclusion of PT/Cy in HSCT conditioning has been associated with a particularly high degree of $\mathrm{HC}(10,12)$. PT/Cy may exacerbate bleeding predisposition by inflicting substantial damage to the urothelium of a robustly immunosuppressed and immunocompromised host. In our patient, BKVassociated bleeding may have been suggestive of a propensity for endothelial damage already underway, with late-onset VOD/ SOS developing consequently.

It is unclear if our patient's bleeding complications were associated with high BK viremia post-HSCT, an unusual manifestation of BKV nephropathy $(13,14)$, or were exacerbated by an underlying immunodeficiency-related vasculopathy. Postmortem analysis did not show evidence of renal polyomavirus, but limited sampling not representative of cross-sectional involvement or false negativity may have precluded the ability to detect BKV (15).

Renal arteriography performed to control left renal hemorrhage demonstrated findings suggestive of vasculopathy 


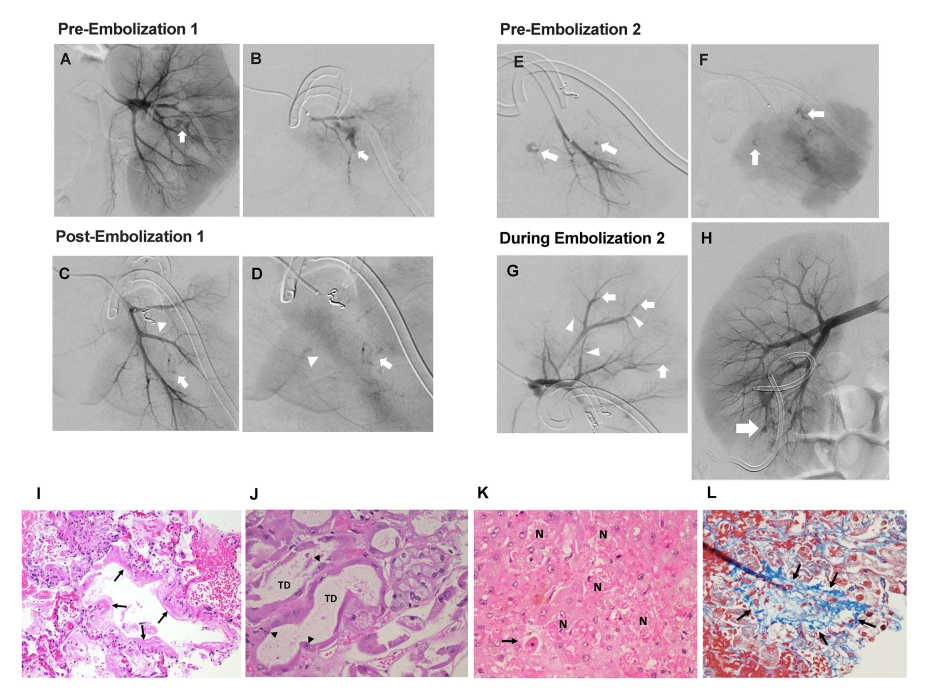

FIGURE 2 | Interventional radiology findings. (A) Selective left renal arteriogram demonstrating active arterial bleeding in the midpole area (white arrow). (B) Superselective left renal arteriogram confirming active bleeding source (white arrow). (C) Superselective left renal arteriogram showing coil occlusion of arterial bleeding source (white arrowhead), as well as suspicious second bleeding source (white arrow). (D) Superselective left renal arteriogram (parenchymal phase) showing slower extravasation from same location as image C (white arrow), as well as third potential bleeding source (white arrowhead). (E) Superselective left renal arteriogram performed 72 hours after embolization redemonstrating bleeding sources (white arrows) in early arterial phase and delayed (F) images. (G) Superselective left upper pole renal arteriogram performed during second embolization showing distal vessel narrowing (white arrowheads) and occlusions (white arrows). (H) Right renal arteriogram performed during second left renal embolization showing a lower pole arteriovenous fistula (white arrow). Pathology findings. (I) Exudative phase diffuse alveolar damage with hyaline membrane formation (arrows) (H\&E, 200x). (J) Acute tubular injury with tubular dilation (TD) and reactive atypia of surviving tubular epithelial cells (arrowheads) (H\&E, 400x). (K) Hepatic necrosis (N) and apoptosis (arrow), (H\&E, 400x). (L) Occluded hepatic vein filled with loose blue-stained collagen (arrows) (Masson trichrome, 400x).

including irregular luminal contour, peripheral branch occlusions, and arterial extravasation. DOCK8 deficiency has been associated with vasculitis leading to aortic aneurysm, calcification, or stroke $(7,16-18)$. Vascular changes noted at arteriography showed distribution at the distal arterial branches and arcuate arteries in the left kidney, which more closely corresponds with vessels affected by immune complex small vessel vasculopathies. Although vasculitis could be associated with hematologic malignancy (DLBCL), viral infection, or DOCK8 mutation, specific attribution is speculative at best.

Management of $\mathrm{BK}$ viremia and $\mathrm{BKV}-\mathrm{HC}$ with current supportive care measures, including cidofovir, has shown only suboptimal benefit $(19,20)$. Given limited options for BKV-HC treatment, anti-BK VSTs address a critical vulnerability in patients with poor or absent T-cell mediated immunity and are feasible for posttransplant use. Using HSCT-donor derived VSTs, Nelson et al. found that $87 \%$ of patients with BK viremia and/or $\mathrm{HC}(\mathrm{n}=38)$ responded to $\geq 1$ anti-BK VST infusions; 58\% completely cleared BKV and demonstrated resolution of $\mathrm{HC}$ symptoms $\leq$ grade II (10). Olson et al. describe comparable outcomes with HLA-matched third-party anti-BK VSTs (21). Infusion-related adverse events were rare with HSCT-donor derived or third-party VSTs $(10,21)$.

Toxicity concerns with allogeneic adoptive T-cell transfer include GVHD and CRS (22). One study of donor-derived and third-party “off-the-shelf” VSTs reported mild GVHD in 11\% of patients with primary immunodeficiencies, with all cases suspected to be transplant-related rather than VST induced
(23). CRS has been recorded in $\leq 2 \%$ of patients receiving VSTs (24). Our patient, however, developed CRS requiring significant support after infusion with the same VST product he previously tolerated. High viral load and disseminated disease have been suggested to increase risk of developing CRS post-VST infusion (25). Interestingly, in the days preceding post-HSCT VST infusion, our patient had a rising CRP. This was potentially associated with life-threatening hematuria and repeated renal interventions, as no obvious alternative infectious source was identified. We postulate that this pre-existing inflammatory milieu in the context of high BK viremia and donor chimerism (100\%), with more reactive lymphocytes than in the pre-HSCT setting, likely all converged and collectively contributed to this severe degree of CRS.

$\mathrm{BK}$ viremia and $\mathrm{BKV}-\mathrm{HC}$ can cause life-threatening complications following HSCT, particularly in those with immunodeficiency. Early HSCT consideration may improve outcomes in patients with DOCK8 deficiency, especially if HSCT can be performed prior to the development of comorbidities associated with the underlying disease which may complicate the transplant course. While proceeding to HSCT with active viremia and sequelae thereof is challenging, HSCT may also be the only path forward in those unable to fully clear their viral disease without immune reconstitution. Thus, management of underlying comorbidities should be optimized as feasible. The use of VSTs in the pre-HSCT setting in our case served to optimize control of BKV and associated BKV-HC prior to HSCT. Use of $\mathrm{PT} / \mathrm{Cy}$ also requires careful monitoring, 
particularly with inclusion of cyclophosphamide in HSCT conditioning and cumulative $\mathrm{HC}$ risk. This case raises awareness of severe complications of BKV-HC. Further study of optimal management strategies of viral disease and severe $\mathrm{BKV}-\mathrm{HC}$ posttransplant is needed.

\section{DATA AVAILABILITY STATEMENT}

The original contributions presented in the study are included in the article/supplementary material. Further inquiries can be directed to Nirali.Shah@nih.gov.

\section{ETHICS STATEMENT}

The studies involving human participants were reviewed and approved by National Cancer Institute, Institutional Review Board. Written informed consent to participate in this study was provided by the participants' legal guardian/next of kin.

\section{REFERENCES}

1. Aydin SE, Kilic SS, Aytekin C, Kumar A, Porras O, Kainulainen L, et al. DOCK8 Deficiency: Clinical and Immunological Phenotype and Treatment Options - A Review of 136 Patients. J Clin Immunol (2015) 35(2):189-98. doi: 10.1007/s10875-014-0126-0

2. Cuellar-Rodriguez J, Freeman AF, Grossman J, Su H, Parta M, Murdock H, et al. Matched Related and Unrelated Donor Hematopoietic Stem Cell Transplantation for DOCK8 Deficiency. Biol Blood Marrow Transplant (2015) 21(6):1037-45. doi: 10.1016/j.bbmt.2015.01.022

3. Al-Herz W, Chu JI, van der Spek J, Raghupathy R, Massaad MJ, Keles S, et al. Hematopoietic Stem Cell Transplantation Outcomes for 11 Patients With Dedicator of Cytokinesis 8 Deficiency. J Allergy Clin Immunol (2016) 138852 859(3):e853. doi: 10.1016/j.jaci.2016.02.022

4. Aydin SE, Freeman AF, Al-Herz W, Al-Mousa HA, Arnaout RK, Aydin RC, et al. Hematopoietic Stem Cell Transplantation as Treatment for Patients With DOCK8 Deficiency. J Allergy Clin Immunol Pract (2019) 7(3):848-55. doi: 10.1016/j.jaip.2018.10.035

5. Pillay BA, Avery DT, Smart JM, Cole T, Choo S, Chan D, et al. Hematopoietic Stem Cell Transplant Effectively Rescues Lymphocyte Differentiation and Function in DOCK8-Deficient Patients. JCI Insight (2019) 4(11):e127527. doi: $10.1172 /$ jci.insight.127527

6. Zhang Q, Davis JC, Lamborn IT, Freeman AF, Jing H, Favreau AJ, et al. Combined Immunodeficiency Associated With DOCK8 Mutations. N Engl J Med (2009) 361(21):2046-55. doi: 10.1056/NEJMoa0905506

7. Su HC, Jing H, Angelus $P$, Freeman AF. Insights Into Immunity From Clinical and Basic Science Studies of DOCK8 Immunodeficiency Syndrome. Immunol Rev (2019) 287(1):9-19. doi: 10.1111/imr.12723

8. Patte C, Auperin A, Gerrard M, Michon J, Pinkerton R, Sposto R, et al. Results of the Randomized International FAB/LMB96 Trial for Intermediate Risk BCell non-Hodgkin Lymphoma in Children and Adolescents: It Is Possible to Reduce Treatment for the Early Responding Patients. Blood (2007) 109 (7):2773-80. doi: 10.1182/blood-2006-07-036673

9. Goldman S, Smith L, Anderson JR, Perkins S, Harrison L, Geyer MB, et al. Rituximab and FAB/LMB 96 Chemotherapy in Children With Stage III/IV BCell non-Hodgkin Lymphoma: A Children's Oncology Group Report. Leukemia (2013) 27(5):1174-7. doi: 10.1038/leu.2012.255

10. Nelson AS, Heyenbruch D, Rubinstein JD, Sabulski A, Jodele S, Thomas S, et al. Virus-Specific T-Cell Therapy to Treat BK Polyomavirus Infection in Bone Marrow and Solid Organ Transplant Recipients. Blood Adv (2020) 4 (22):5745-54. doi: 10.1182/bloodadvances.2020003073

\section{AUTHOR CONTRIBUTIONS}

EMH and NNS wrote the first version of the manuscript. CG, EL, VAV, HC, JK-S, BY, CH, HD, HS, DDH, HCS, MG, AFF, NNS provided direct patient care and/or guidance on management. DEK provided pathology review. All authors contributed to the final version of the manuscript.

\section{ACKNOWLEDGMENTS}

The authors gratefully acknowledge the study participants reported on in this case (family provided consent for publication), and the faculty and staff and care providers at the National Institutes of Health Clinical Center, Children's National Medical Center, and Cincinnati Children's Hospital who provided their support and expertise in the care of this patient. This work was supported in part by the Intramural Research Program, Center of Cancer Research, National Cancer Institute, National Institute of Allergy and Infectious Diseases, and NIH Clinical Center, National Institutes of Health (ZIA BC 011823).

11. Shah NN, Freeman AF, Su H, Cole K, Parta M, Moutsopoulos NM, et al. Haploidentical Related Donor Hematopoietic Stem Cell Transplantation for Dedicator-Of-Cytokinesis 8 Deficiency Using Post-Transplantation Cyclophosphamide. Biol Blood Marrow Transplant (2017) 23(6):980-90. doi: 10.1016/j.bbmt.2017.03.016

12. Harkensee C, Vasdev N, Gennery AR, Willetts IE, Taylor C. Prevention and Management of BK-Virus Associated Haemorrhagic Cystitis in Children Following Haematopoietic Stem Cell Transplantation-a Systematic Review and Evidence-Based Guidance for Clinical Management. $\mathrm{Br}$ J Haematol (2008) 142(5):717-31. doi: 10.1111/j.1365-2141.2008.07254.x

13. Vigil D, Konstantinov NK, Barry M, Harford AM, Servilla KS, Kim YH, et al. BK Nephropathy in the Native Kidneys of Patients With Organ Transplants: Clinical Spectrum of BK Infection. World J Transplant (2016) 6(3):472-504. doi: 10.5500/wjt.v6.i3.472

14. Shah A, Kumar V, Palmer MB, Trofe-Clark J, Laskin B, Sawinski D, et al. Native Kidney BK Virus Nephropathy, a Systematic Review. Transpl Infect Dis (2019) 21(4):e13083. doi: 10.1111/tid.13083

15. Wiesend WN, Parasuraman R, Li W, Farinola MA, Rooney MT, Hick SK, et al. Adjuvant Role of P53 Immunostaining in Detecting BK Viral Infection in Renal Allograft Biopsies. Ann Clin Lab Sci (2010) 40(4):324-9.

16. Petrogiannis-Haliotis T, Sakoulas G, Kirby J, Koralnik IJ, Dvorak AM, MonahanEarley R, et al. BK-Related Polyomavirus Vasculopathy in a Renal-Transplant Recipient. N Engl J Med (2001) 345(17):1250-5. doi: 10.1056/NEJMoa010319

17. Sabry A, Hauk PJ, Jing H, Su HC, Stence NV, Mirsky DM, et al. Vaccine Strain Varicella-Zoster Virus-Induced Central Nervous System Vasculopathy as the Presenting Feature of DOCK8 Deficiency. J Allergy Clin Immunol (2014) 133 (4):1225-7. doi: 10.1016/j.jaci.2013.11.031

18. AlKhater SA. CNS Vasculitis and Stroke as a Complication of DOCK8 Deficiency: A Case Report. BMC Neurol (2016) 16:54. doi: 10.1186/s12883-016-0578-3

19. Foster JH, Cheng WS, Nguyen NY, Krance R, Martinez C. Intravesicular Cidofovir for BK Hemorrhagic Cystitis in Pediatric Patients After Hematopoietic Stem Cell Transplant. Pediatr Transplant (2018) 22(3): e13141. doi: 10.1111/petr.13141

20. Laskin BL, Denburg MR, Furth SL, Moatz T, Altrich M, Kleiboeker S, et al. The Natural History of BK Polyomavirus and the Host Immune Response After Stem Cell Transplantation. Clin Infect Dis (2019) 71:3044-54. doi: $10.1093 / \mathrm{cid} / \operatorname{ciz} 1194$

21. Olson A, Lin R, Marin D, Rafei H, Bdaiwi MH, Thall PF, et al. Third-Party BK Virus-Specific Cytotoxic T Lymphocyte Therapy for Hemorrhagic Cystitis Following Allotransplantation. J Clin Oncol (2021) 39(24):2710-9. doi: 10.1200/JCO.20.02608 
22. Fajgenbaum DC, June CH. Cytokine Storm. N Engl J Med (2020) 383 (23):2255-73. doi: 10.1056/NEJMra2026131

23. Naik S, Nicholas SK, Martinez CA, Leen AM, Hanley PJ, Gottschalk SM, et al. Adoptive Immunotherapy for Primary Immunodeficiency Disorders With Virus-Specific T Lymphocytes. J Allergy Clin Immunol (2016) 1371498-1505 (5):e1491. doi: 10.1016/j.jaci.2015.12.1311

24. Keller MD, Bollard CM. Virus-Specific T-Cell Therapies for Patients With Primary Immune Deficiency. Blood (2020) 135(9):620-8. doi: 10.1182/ blood.2019000924

25. Cohen JM, Cooper N, Chakrabarti S, Thomson K, Samarasinghe S, Cubitt D, et al. EBV-Related Disease Following Haematopoietic Stem Cell Transplantation With Reduced Intensity Conditioning. Leuk Lymphoma (2007) 48(2):256-69. doi: 10.1080/10428190601059837

Author Disclaimer: The content of this publication does not necessarily reflect the views of policies of the Department of Health and Human Services, nor does mention of trade names, commercial products, or organizations imply endorsement by the U.S. Government.
Conflict of Interest: The authors declare that the research was conducted in the absence of any commercial or financial relationships that could be construed as a potential conflict of interest.

Publisher's Note: All claims expressed in this article are solely those of the authors and do not necessarily represent those of their affiliated organizations, or those of the publisher, the editors and the reviewers. Any product that may be evaluated in this article, or claim that may be made by its manufacturer, is not guaranteed or endorsed by the publisher.

Copyright (c) 2021 Holland, Gonzalez, Levy, Valera, Chalfin, Klicka-Skeels, Yates, Kleiner, Hadigan, Dave, Shalabi, Hickstein, Su, Grimley, Freeman and Shah. This is an open-access article distributed under the terms of the Creative Commons Attribution License (CC BY). The use, distribution or reproduction in other forums is permitted, provided the original author(s) and the copyright owner(s) are credited and that the original publication in this journal is cited, in accordance with accepted academic practice. No use, distribution or reproduction is permitted which does not comply with these terms. 